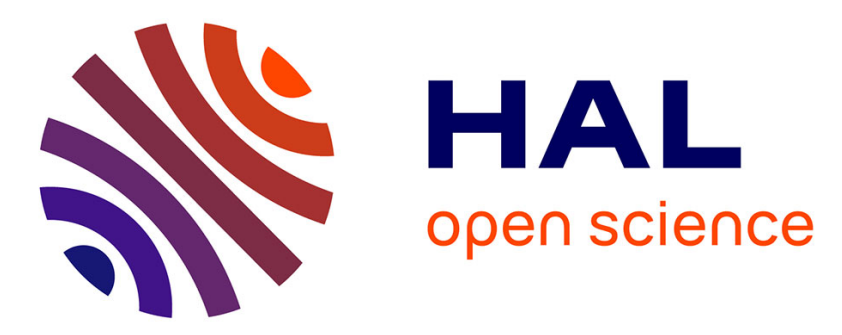

\title{
Why VCG auctions can hardly be applied to the pricing of inter-domain and ad hoc networks
}

\author{
Patrick Maillé, Bruno Tuffin
}

\section{To cite this version:}

Patrick Maillé, Bruno Tuffin. Why VCG auctions can hardly be applied to the pricing of inter-domain and ad hoc networks. 3rd EuroNGI conference on Next Generation Internet Networks, May 2007, Trondheim, Norway. pp.36 - 39, 10.1109/NGI.2007.371195 . hal-02164968

\section{HAL Id: hal-02164968 \\ https://hal.science/hal-02164968}

Submitted on 25 Jun 2019

HAL is a multi-disciplinary open access archive for the deposit and dissemination of scientific research documents, whether they are published or not. The documents may come from teaching and research institutions in France or abroad, or from public or private research centers.
L'archive ouverte pluridisciplinaire HAL, est destinée au dépôt et à la diffusion de documents scientifiques de niveau recherche, publiés ou non, émanant des établissements d'enseignement et de recherche français ou étrangers, des laboratoires publics ou privés. 


\section{Why VCG auctions can hardly be applied to the pricing of inter-domain and ad hoc networks}

\author{
Patrick Maillé \\ GET/ENST Bretagne \\ 2, rue de la Châtaigneraie - CS 17607 \\ 35576 Cesson-Sévigné Cedex - FRANCE \\ Email: patrick.maille@enst-bretagne.fr
}

\author{
Bruno Tuffin \\ IRISA-INRIA \\ Campus de Beaulieu \\ 35042 Rennes Cedex - FRANCE \\ Email: btuffin@irisa.fr
}

\begin{abstract}
The use of Vickrey-Clarke-Groves (VCG) auction mechanisms is gaining popularity in the networking community, where it seems compulsory to incentivize selfish nodes (in ad hoc networks) or domains (in inter-domain communications) to forward the traffic of their peers. Indeed, VCG auctions are known to both be efficient and produce proper incentives.

In this note, we argue that, in fact, VCG auctions can hardly be applied to those problems, for different reasons depending on the model studied:

- if some resource constraints (bandwidth, spectrum, and/or power) have to be taken into account, then computing allocations and prices implies solving optimization problems that are computationally hard for general network topologies.

- If there are no such resource constraints, then VCG auctions, even if verifying many important and satisfactory properties, cannot verify a major one that is budget balance: the sum of subsidies given to relay nodes exceeds the sum of charges paid by traffic senders. This means that the auction regulator is required to continuously inject money to make the scheme work, which is unlikely to happen.
\end{abstract}

In a second step, we discuss the combinations of properties that can be verified together, and prioritize them for finding out a proper pricing scheme.

\section{INTRODUCTION}

The Internet has become a commercial and competitive network with many service providers. Those providers need to exchange traffic to ensure end-to-end delivery, which requires peering agreements so that go-between domains accept to forward traffic. Very similar problems occur in ad hoc networks where nodes, the terminals, are assumed to forward the traffic of others at the expense of their own battery power.

Pricing appears a promising way to introduce incentives for forwarding traffic in both of those contexts. Vickrey-ClarkeGroves (VCG) auctions especially have recently received a lot of attention since they are known to yield efficient outcomes, with enjoyable properties such as incentive compatibility, meaning that players' best interest is to reveal their true valuation of the proposed service, or individual rationality, meaning that players will always benefit from entering the auction.

Here, we aim at showing that, actually, VCG auctions are difficult to implement in inter-domain or ad hoc networks. While they enjoy the incentive compatibility, individual rationality, and efficiency properties, they present at least one prohibitive drawback: either they need a computationally difficult optimization problem to be solved, or they require that the auction manager insert money into the game since the sum of payments from those sending (or receiving) traffic is always less than the sum of awards distributed to those forwarding. This last problem is not only verified on the specific problems of pricing inter-domain and ad hoc networks, but has been shown in the literature in a general setting, making it irrelevant to try and get budget balance by modifying the original models.

This situation of non-balance seems to us likely to deter from implementation. We therefore further discuss the properties that a pricing scheme should enjoy, try to prioritize them, and look at the possible combinations, in order to provide hints on the families of pricing schemes that should be dealt with.

Note that this kind of problem has recently received some attention in the economics community. Indeed, in [1], it is shown that when preferences are quasi-linear, an incentive compatible mechanism can be efficient or can balance the budget, but it cannot do both at the same time. The authors then characterize a class of budget balanced and incentive compatible mechanisms, and single out the one with the smallest welfare loss (based on the Shapley value formula). They also conversely look at mechanisms which are efficient and not balanced budget. Though, the context of that work is slightly different from ours, since their problem consists in sharing the cost of a given service among users, where the cost is a submodular function of the set of receiving service, and where users cannot be partially served. The objective function is therefore different: there is no allocation rate involved, and there is no real focus on a network topology.

\section{PRinciples AND PROPERTIES OF VCG AUCTIONS}

Again, pricing has been seen as a means of interest to introduce incentives in situations where each new user introduces an added-value to the network capability; interdomain routing and ad hoc networks being typical areas of applications. Indeed, a very basic idea would be for instance to extend the current Border Gateway Protocol (BGP) to allow interdomain routing. The extension would consist in introducing pricing such that each intermediate provider would receive a financial incentive for accepting to forward packets 
of concurrent providers. It is exactly the same problem in ad hoc networks where forwarding packets of neighbors is at the expense of limited battery capacities, and therefore needs to be compensated in some way. VCG auctions have been regarded as one of the most prevalent ways to introduce those rewarding issues. In all those applications, each player (a domain in case of interdomain routing or a node of the ad hoc network) tries to maximize its own benefits, that is the valuation for sending its own traffic (depending on the volume of traffic he sends), plus the amount of money gained from the forwarded traffic, minus the cost of sending traffic (financial cost plus engineering cost, in terms of battery for instance).

Vickrey-Clarke-Groves auctions [2], [3], [4] apply to any problem where players (users) have a quasi-linear utility function. This means that the utility $U_{i}$ of each user $i$ depends on the outcome (say, the resource allocation vector) $a$ and on the price $c_{i}$ he is charged (notice that $c_{i}$ can be non-positive when $i$ is subsidized), and can be written

$$
U_{i}\left(a, c_{i}\right)=\theta_{i}(a)-c_{i}
$$

where $\theta_{i}$ is called the valuation or willingness-to-pay function of user $i$.

VCG auctions work as follows:

1) each user is asked to reveal his valuation function (let us denote by $\tilde{\theta}_{i}$ the function declared by user $i$ with real valuation function $\theta_{i}$ );

2) the mechanism computes an outcome $a(\tilde{\theta})$ that maximizes the declared social welfare ${ }^{1}$ :

$$
a(\tilde{\theta}) \in \arg \max _{x} \sum_{i} \tilde{\theta}_{i}(x)
$$

3) the price paid by each user corresponds to the loss of declared welfare he imposes to the others through his presence:

$$
c_{i}=\max _{x} \sum_{j \neq i} \tilde{\theta}_{j}(x)-\sum_{j \neq i} \tilde{\theta}_{j}(a(\tilde{\theta})) .
$$

Then the mechanism verifies three major properties:

- Incentive compatibility: for each user, bidding truthfully (i.e. declaring $\tilde{\theta}_{i}=\theta_{i}$ ) is a dominant strategy.

- Individual rationality: each truthful player obtains a nonnegative utility.

- Efficiency: when players bid truthfully, social welfare $\left(\sum_{i} \theta_{i}\right)$ is maximized.

\section{VCG AUCTIONS IN INTER-DOMAIN OR AD HOC NETWORKS}

In networks, the form of each agent $i$ 's valuation function $\theta_{i}$ can be precised:

- traffic initiators (senders) are accepting to pay up to a threshold to obtain a given transmission rate from one point to another: therefore their valuation function depends only on their own resource allocation. Depending

\footnotetext{
${ }^{1}$ The maximization is over the set of feasible outcomes, that may be limited by resource constraints.
}

on the type of flow, the individual demand of a sender can be elastic (that is tolerant to variations in the obtained service) or inelastic (that is with strict requirements).

- Intermediate nodes or domains have a negative valuation function, that reflects their perceived costs for transferring traffic. Generally, those costs depend on the total amount of traffic that has to be transferred, or (in ad hoc networks using power control) on the total power needed to transfer that traffic.

The goals of introducing VCG auctions in inter-domain or ad hoc networks is to obtain an efficient routing (by enforcing cooperation) and to incentivize participation for transferring traffic. The rules are designed such that the senders (or receivers) pay for the traffic that is carried and the intermediate nodes are subsidized for transferring traffic, compensating their transmission costs. The amount to be awarded is defined by the specific pricing rule (2) employed.

In the following, we argue that VCG auctions applied to such networks present at least one prohibitive drawback, large computational complexity and/or negative budget balance, depending on whether resource constraints are taken into account when determining the outcome.

A. Networks with resource constraints: VCG and computational complexity

When communication links are not overdimensioned (which is particularly true in ad hoc networks where the transmission rates are limited by the radio spectrum, and where interference degrades even more those transmission rates), it may not be possible to satisfy all requests because of congestion. VCG auctions in such contexts would then serve at the same time (i) as an incentive for intermediate nodes to transfer traffic, and (ii) as a rule for selecting the accepted and rejected requests.

Following VCG principles, each potential sender declares his willingness-to-pay function, and each potential intermediate node (i.e. each node, since all node are likely to transfer traffic) declares his cost function. Then allocations are computed in order to maximize the sum of declared valuations (social welfare), that is the sum of prices senders are willing to pay minus the sum of transfer costs (by summing over all senders and intermediate nodes). However, a problem due to the complexity of that computation appears when the set of possible allocations is constrained by resource bounds. More precisely, determining in general the most efficient allocation is a NP-hard problem. As an example, consider a very simple model with only one intermediate node, say $A$, that cannot transfer more than a given amount $Q$ of traffic, and $N$ potential senders with inelastic demand that need the help of $A$ to reach their destination, as represented in Figure 1. The valuation function $\theta_{i}$ of each potential sender $i$ with non-elastic demand is $\theta_{i}\left(a_{i}\right)=\beta_{i} \mathbb{1}_{a_{i} \geq \alpha_{i}}$, which means that sender $i$ is willing to pay $\beta_{i}$ if he is allocated at least $\alpha_{i}$, and 0 otherwise. Then, even if the transferring cost for $A$ is null, maximizing the social welfare $\sum_{i} \theta_{i}\left(a_{i}\right)$ under the constraint $\sum_{i} a_{i} \leq Q$ resumes to solving the knapsack problem, which is known to be NP-hard $[5]$. 


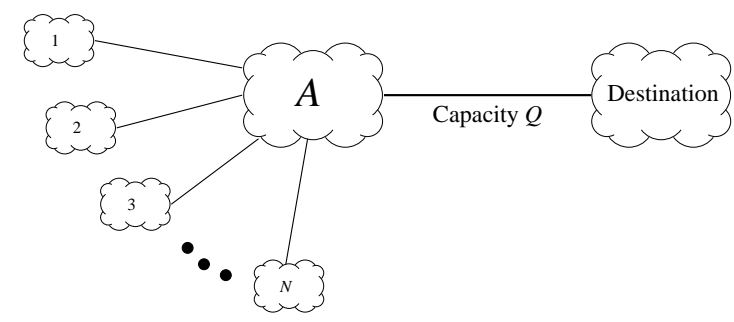

Fig. 1. Allocation problem in case of congestion

In the above example, NP-hardness comes from the inelasticity of demands. For elastic demands, determining the most efficient allocation through both routing and allocations is also NP-hard for non-trivial network topologies when routes have to be single-path [6].

To emphasize on the complexity problems that stem from the application of VCG auctions in networks with capacity constraints, remember that the computation of the price paid (resp. the subsidy received) by each sender (resp. intermediate) needs another optimization problem to be solved (see Equation (2)). Applying VCG auctions with $N$ users therefore implies $N+1$ optimization problems to be solved: those problems must be computationally simple enough.

\section{B. Transfer costs with no resource constraints: VCG and budget balance}

Some networks can be dimensioned such that congestion never occurs. This might be the case for example in core networks, that use the latest technologies and allow high communication rates. It can therefore be assumed that interdomain routing never introduces new constraints due to resource limits. The transfer costs for an intermediate domain are then modelled as being proportional to the amount of traffic to transfer.

Vickrey-Clarke-Groves auctions seem consequently wellsuited to this context, since a request has no effect on another one, and maximizing the overall social welfare can then be done by performing independently a least-cost path search for each request, removing the complexity issue of previous subsection.

This kind of model is assumed in [7], [8], where VCG auctions are used to produce the incentives to transfer traffic. The model consists of $n$ domains (or autonomous systems), numbered from 1 to $n$. Inter-domain routing is handled by a simple modification of the Border Gateway Protocol (BGP) [7]. It is assumed that the amount of traffic is $T_{i j}$ from domain $i$ to domain $j$, and that the per-packet transit cost is $c_{k}$ for domain $k(1 \leq k \leq n)$. Valuation of intermediate domain $k$ for a given allocation (here, a routing decision) is thus

$$
\theta_{k}(\text { routing })=-c_{k} \sum_{\{(i, j) \text { routed trough } k\}} T_{i j} .
$$

Each domain is asked to declare its transit $\cos t c_{k}$, and the least (declared) cost route path $(i, j)$ is computed for each origindestination pair $(i, j)$. Since there are no resource constraint, those routes minimize the total (declared) cost

$$
\sum_{i, j} T_{i j} \sum_{k \in \operatorname{path}(i, j)} c_{k}
$$

which amounts to the maximization problem (1) when using (3). Equivalently, the computation of VCG subsidies for each domain $k$ can be done per each origin-destination pair $(i, j)$, by applying the pricing rule (2) to the problem of routing only the traffic from $i$ to $j$. Those subsidies ensure that intermediate domains, acting selfishly, truthfully reveal their transfer costs, and therefore that the routing is efficient.

Nevertheless, one question not addressed in [7], [8] is: who pays the subsidies? It is natural to think that the origin ${ }^{2}$ should pay them, but would that be suitable if the sum of subsidies equals $10 \$$ while the sender is willing to pay only $1 \$$ for his traffic to reach destination?

Actually, an efficient scheme should take into account the willingness-to-pay of the traffic sender to determine whether it is socially optimal for that traffic to be carried (which is the case if and only if the sender valuation for the traffic exceeds the sum of tranfer costs of the intermediate domains). VCG auctions fit perfectly with that case: just consider the traffic sender as a player that is asked to declare the maximum price he is willing to pay. Then because of social welfare maximization (1), that traffic will only be sent if it socially profitable, and the pricing rule (2) will determine a price to pay for the sender such that truthfully declaring his willingnessto-pay is a dominant strategy.

The same kind of model is presented by Anderegg and Eidenbenz [9] for incentivizing cooperation in ad hoc networks, with the same drawbacks as in [7], [8]. Basically, transfer costs depend here on the minimum power required to reach the next neighbour. The model therefore generalizes the one in [7] by associating several costs to each node, one per neighbour, instead of just one. The analysis and results are identical though. The sender's willingness-to-pay is (again) not taken into account, but it is assumed that the sender should pay the total declared transfer cost. Though, for a VCG auction where both intermediates and senders are treated as selfish players, the VCG price that the sender should pay according to (2) is the sum of declared costs if the traffic is effectively sent. However, as highlighted in [9], this price is always below the sum of subsidies. That problem of non budget balance is defined as the overpayment; a relative bound with respect to what is paid is provided. The balance of the mechanism is therefore always negative, which means that money has to be inserted to make the scheme work.

The model in [9] is modified by Eidenbenz et al in [10]: subsidies are still computed according to (2), but the sender is charged the total declared cost of a "second least-cost path", i.e. the least-cost path with all nodes in the cost-efficient path

\footnotetext{
${ }^{2}$ Or the termination for some applications like file downloading. Since those considerations do not change the reasoning, we assume in the following that the traffic sender is charged.
} 
removed $^{3}$, if that charge is below his declared willingness-topay. The authors argue that such charges are closer to the sum of subsidies than VCG charges, and that the receiver should compensate the difference to reach budget balance (from which the scheme is closer). The scheme is also shown to be incentive compatible. Nevertheless, that mechanism is not efficient anymore, since situations may occur where a request is rejected whereas it would maximize social welfare (this is the case when the sender's valuation is between the total costs of the first and the second least-cost paths). Therefore, introducing budget balance results here in a loss of efficiency.

In the next subsection, we review general results about auctions that give some hints about the properties we can expect from a properly designed mechanism.

\section{General results on auctions}

Green and Laffont [11] establish that when players have quasi-linear utility functions, every allocation and pricing mechanism that is simultaneously efficient and incentive compatible is a Groves mechanism, VCG auctions being a particular case of them.

More recently, Krishna and Perry [12] prove that among the mechanisms that are efficient, Bayesian incentive compatible ${ }^{4}$, and Bayesian individually rational, VCG prices maximize the expected price paid by each agent (that can be negative). Since incentive compatibility (resp. individual rationality) respectively imply Bayesian incentive compatibility (resp. individual rationality), VCG auctions minimize the overpayment (always existing without capacity constraints; otherwise, it may amount to maximizing the benefits, if any) among the schemes that verify the three properties: incentive compatibility, individual raionality and efficiency. The fact that the introduction of capacity constraints may result in no overpayment stems from the appearance of congestion charges for the sender that can overtake the overpayment.

Therefore, for a network without resource constraints as studied in subsection III-B, the negative balance of VCG auctions imply that the four properties

- incentive compatibility,

- efficiency,

- individual rationality,

- budget balance,

cannot hold simultaneously.

\footnotetext{
${ }^{3}$ For that price to be defined, the authors assume that every pair $(s, t)$ of nodes is 2-connected, meaning that there exist two node-disjoint paths connecting $(s, t)$

${ }^{4}$ In [12], Krishna and Perry take a Bayesian approach: they consider that valuation functions are distributed according to a given probability distribution, and that each player, knowing his own valuation, derives a conditional distribution on the valuation of the others. Bayesian incentive compatibility means here that playing truthfully ensures each player to maximize his expected utility conditionally on his valuation. Moreover, the scheme is Bayesian individually rational if that conditional expected utility is non-negative.
}

\section{DISCUSSION: WHICH COMBINATION OF PROPERTIES TO PREFER?}

This paper focused on the applicability of VCG auctions in inter-domain and ad hoc networks. We have exhibited prohibitive disadvantages of that scheme, related to the computational complexity involved and/or the financial cost of the mechanism.

Consequently, a pricing scheme adapted to that context must relax at least one of the four properties given in subsection IIIC. It seems to us that budget balance cannot be relaxed, since such a mechanism would not be economically viable. Second, we think that individual rationality is also compulsory: users may be unwilling to risk having a negative utility and would perceive the scheme badly. Moreover, the revelation principle [13] states that the outcome of any auction game is reachable by an incentive compatible mechanism, which implies that incentive compatibility is always reachable.

The only property that remains to be relaxed is efficiency (notice that this property is relaxed in [10], but budget balance is not assured either in that work). However, it is still desirable that the allocation remain close to the social optimum, thus a direction for future work in that context could be to design a scheme with bounded inefficiency.

\section{ACKNOWLEDGEMENT}

The authors acknowledge the partial support of EuroNGI NoE through the AUCTIONS project, and the anonymous referees for pointing out the work of Moulin and Shenker [1].

\section{REFERENCES}

[1] H. Moulin and S. Shenker, "Strategyproof sharing of submodular costs: Budget balance versus efficiency," Economic Theory, vol. 18, pp. 511533, 2001.

[2] W. Vickrey, "Counterspeculation, auctions, and competitive sealed tenders," Journal of Finance, vol. 16, no. 1, pp. 8-37, Mar 1961.

[3] E. H. Clarke, "Multipart pricing of public goods," Public Choice, vol. 11, pp. 17-33, 1971.

[4] T. Groves, "Incentives in teams," Econometrica, vol. 41, no. 3, pp. 617631, Jul 1973.

[5] G. J. Woeginger, "Exact algorithms for NP-hard problems: A survey," in Lecture Notes in Computer Science, ser. 2570, 2003, pp. 185-207.

[6] J. Wang, L. Li, S. H. Low, and J. C. Doyle, "Can shortest-path routing and tcp maximize utility," in Proc. of IEEE INFOCOM, 2003.

[7] J. Feigenbaum, C. Papadimitriou, R. Sami, and S. Shenker, "A BGPbased mechanism for lowest-cost routing," in Proc. of the 21st ACM Symposium on Principles of Distributed Computing, 2002.

[8] J. Hershberger and S. Suri, "Vickrey prices and shortest paths: What is an edge worth?" in Proc. of the 42nd IEEE Symposium on Foundations of Computer Science, 2001, pp. 252-259.

[9] L. Anderegg and S. Eidenbenz, "Ad hoc-VCG: A truthful and costefficient routing protocol for mobile ad hoc networks with selfish agents," in Proc. of 9th annual International Conference on Mobile Computing and Networking (MobiCom 2003), San Diego, CA, USA, Sept 2003, pp. 245-259.

[10] S. Eidenbenz, P. Santi, and G. Resta, "COMMIT: A sender-centric truthful and energy-efficient routing protocol for ad hoc networks," in Proc. of 5th IEEE Intl Workshop on Algorithms for Wireless, Mobile, Ad Hoc and Sensor Networks (WMAN 05), Apr 2005.

[11] J. Green and J.-J. Laffont, "Characterization of satisfactory mechanisms for the revelation of preferences for public goods," Econometrica, vol. 45, no. 2, pp. 427-438, Mar 1977.

[12] V. Krishna and M. Perry, "Efficient mechanism design," Pennsylvania State University, Tech. Rep., 1998.

[13] R. B. Myerson, "Optimal auction design," Mathematics of Operations Research, vol. 6, no. 1, pp. 58-73, Feb 1981. 